\title{
Erratum: Observation of femtosecond infrared luminescence in gold [Phys. Rev. B 100, 125405 (2019)]
}

\author{
Tohru Suemoto $\odot$, Ken-ichi Yamanaka, and Noriaki Sugimoto
}

(Received 31 August 2021; published 13 September 2021)

DOI: 10.1103/PhysRevB.104.119905

We report an error in the equation used for fitting the time-resolved luminescence spectra.

The coefficient $E_{\mathrm{L}}^{3}$ in Eq. (1) should be replaced by $E_{\mathrm{L}}$. A correct expression for similar formulation is found in the Supplemental Material of Ref. [1]. The revised equation for the luminescence intensity at $E_{\mathrm{L}}$ in units of photons/s per unit energy window is as follows:

$$
L\left(E_{\mathrm{L}}\right)=\varepsilon\left(E_{e x c}\right) \varepsilon\left(E_{\mathrm{L}}\right) W E_{\mathrm{L}} \int_{-\infty}^{\infty} f(x)\left[1-f\left(x-E_{\mathrm{L}}\right)\right] D(x) D\left(x-E_{\mathrm{L}}\right) d x,
$$

where $\varepsilon\left(E_{\mathrm{L}}\right)$ is the emissivity at $E_{\mathrm{L}}$ and $\varepsilon\left(E_{\text {exc }}\right)$ is the absorption at the excitation photon energy $E_{\text {exc }}$, which is equivalent to the emissivity according to Kirchhoff's law of thermal radiation. The radiative recombination rate at $E_{\mathrm{L}}$ is mainly determined by the energy integral in Eq. (1), i.e., the convolution of the number of the occupied electrons $f(x) D(x)$ and the unoccupied electrons [1-f(x-E $\left.\left.E_{L}\right)\right] D\left(x-E_{L}\right)$, where $D(x)$ and $f(x)$ are the density of states and the electron distribution function consisting of thermal and nonthermal components, respectively. Although the factor $W$ is determined by the transition matrix element, which depends generally on kinetic energy and the wave number of the contributing electrons, we assume $W$ is constant. A more detailed discussion on the radiative recombination rate and the dynamics of electron relaxation processes are found in Ref. [2].

In analyzing the spectral shape, we assumed photon energy independent emissivity in that paper. However, it has been found inappropriate afterwards, that is, the emissivity decreases considerably at lower energy as described in our recent paper [3]. Therefore, we did not try to use the revised formula for the original experimental data. Instead, we performed fitting with this formula for the emissivity corrected spectra, which are regarded as internal spectra. The fitting results and revised parameters are found in Figs. 2(a) and 8 of Ref. [3]. The main physics and the conclusions are not changed by this revision.

We are grateful to Dr. S. Ono for pointing out the error in the original paper.

[1] T. Haug, P. Klemm, S. Bange, and J. M. Lupton, Hot-Electron Intraband Luminescence from Single Hot Spots in Noble-Metal Nanoparticle Films, Phys. Rev. Lett. 115, 067403 (2015).

[2] S. Ono and T. Suemoto, Ultrafast photoluminescence in metals: Theory and its application to silver, Phys. Rev. B 102, 024308 (2020).

[3] T. Suemoto, K. Yamanaka, N. Sugimoto, Y. Kobayashi, T. Otsu, S. Tani, and T. Koyama, Relaxation dynamics of hot electrons in the transition metals Au, Ag, Cu, Pt, Pd, and Ni studied by ultrafast luminescence spectroscopy, J. Appl. Phys. 130, 025101 (2021). 